\title{
Impact of adipogenic differentiation on stemness and osteogenic gene expression in extensive culture of human adipose-derived stem cells
}

\author{
Wan Kamarul Zaman Wan Safwani ${ }^{1}$, Suzana Makpol$^{2}$, Somasundaram Sathapan ${ }^{3}$, Kienhui Chua ${ }^{1}$
}

\author{
${ }^{1}$ Department of Physiology, Faculty of Medicine, Universiti Kebangsaan Malaysia, \\ Kuala Lumpur, Malaysia \\ 2Department of Biochemistry, Faculty of Medicine, Universiti Kebangsaan Malaysia, \\ Kuala Lumpur, Malaysia \\ ${ }^{3}$ Subang Jaya Medical Centre, Selangor, Malaysia
}

Submitted: 23 June 2011

Accepted: 9 October 2011

Arch Med Sci 2014; 10, 3: 597-606

DOI: 10.5114 /aoms.2014.43753

Copyright $\odot 2014$ Termedia \& Banach

\section{Abstract}

Introduction: Adipose tissue is a source of multipotent adult stem cells. Most studies on human adipose-derived stem cells (ASC) have been on the early passages. Studies in extensive expansion have not been well established yet. In this study, we aim to investigate the effects of extensive expansion on the adipogenic differentiation capability of ASC.

Material and methods: The ability of ASC to undergo adipogenic differentiation in extensive expansion was evaluated by morphological changes, differentiation assay by using Oil Red $O$ staining and changes in the genes expression levels of adipogenic genes, osteogenic genes and stemness genes using quantitative polymerase chain reaction (qPCR) after induction.

Results: Morphological study showed that the formation of lipid droplets can be observed at all passages but decreased at P20 after induction. Data from $\mathrm{qPCR}$ showed that most adipogenicgenes expression increased significantlyat P5, P10 and P15 but decreased at P20 after induction. On the other hand, osteogenic genes showed no significant changes after adipogenic induction indicating low potentiality of adipogenic-induced ASC to become osteogenic cells. While stemness genes expression levels showed a decrease or no significant changes after adipogenic induction except Nanog3, which showed a significant increase at P15 and P20.

Conclusions: The ability of ASC to differentiate into mature adipogenic cells decreased after P10 and the decrease in the osteogenics gene expression level during adipogenic induction suggested that the osteogenesis and adipogenesis are not parallel events.

Key words: adipose, stem cells, stemness, adipogenesis, osteogenesis, differentiation, culture expansion, cell-based therapy.

\section{Introduction}

Adipose tissue is a source of multipotent adult stem cells and it has been used in tissue engineering as well as in regenerative medicine. The advantages of using adipose tissue as a stem cell source are that it can be obtained in abundance, it has been found to have multilineage differentiation potential and it can be harvested easily with a minimally invasive method

\author{
Corresponding author: \\ Kien-Hui Chua \\ Department of Physiology \\ Faculty of Medicine \\ Universiti Kebangsaan \\ Malaysia \\ Jalan Raja Muda Abdul Aziz \\ 50300 Kuala Lumpur, \\ Malaysia \\ Phone: +603-92897299 \\ Fax: +603-26939687 \\ E-mail: ckienhui@hotmail. \\ com
}


compared to bone marrow. Adipose-derived stem cells (ASC) can differentiate into chondrogenic cells, adipogenic cells, osteogenic cells [1] and neurogenic cells [2]. However, one study reported that bone marrow stem cells (BMSC) have a superior potential for osteogenesis compared to ASC [3]. One of the ideal characteristics of stem cells is that they should be able to maintain their multilineage differentiation ability while they are in long-term culture or undergo extensive subculture. Guilak et al. reported that adipose-derived human mesenchymal stem cells can undergo clonal expansion while maintaining their differentiation ability [4]. However, most of the research on ASC differentiation has been carried out at the early passages (P1-P5) [5, 6].

Adipogenesis is a process which occurs when stem cells differentiate into adipocytes and it happen throughout an organism's lifetime. The function of adipocytes is to store triglycerides during energy excess and mobilise them during deprivation, which is controlled by the lipogenic and lipolytic processes through hormonal signals from the bloodstream [7]. The process of adipogenesis starts with pluripotent mesoderm stem cells. During adipogenic differentiation, committed preadipocytes will withdraw from the cell cycle before undergoing adipose conversion as growth arrest is required for this differentiation [8]. After that, appropriate combination of mitogenic and adipogenic signals is needed to proceed to the next step of the differentiation, which lead to the changes in morphological and biochemical characteristics of the mature adipocyte [9]. There are several genes involved in the adipogenic differentiation. Peroxisome proliferator-activated receptor- $\gamma$ (PPAR) is the main gene in adipogenic differentiation and it maintains adipocyte specific functions such as lipid storage [10]. Lipoprotein lipase (LPL) is involved in lipid metabolism and transport by catalyzing the rate-limiting step in the hydrolysis of the triacylglycerol component [11]. The activation of LPL gene transcription was found to be induced by the PPAR gene in macrophages [12]. Fatty acid binding protein (aP2) plays an important role in the development of metabolic syndrome and the coordination of cholesterol trafficking as well as inflammatory activity $[13,14]$. These genes can be used as markers to study the progression of stem cells in adipogenic differentiation, especially in long-term culture. The quantitative evaluation of these genes is important to determine the threshold level at which the stem cells can differentiate efficiently.

Apart from these genes, stemness genes, e.g. Nanog3 and Sox2, should be studied as well during the progression of stem cell differentiation into other lineages. How these stemness genes affect the process of differentiation, whether their expressions are up-regulated or not, is yet to be established. Our previous study on the effect of long-term culture on ASC showed that the stemness gene expression level decreased from P5 to P15, which indicated that the ability of the cells to differentiate after serial passaging may have decreased as well [15]. Genes involved in osteogenic differentiation, e.g. osteopontin (OSP) and alkaline phosphatase (ALP), should also be studied as osteogenesis shares a similar pathway as adipogenesis. There is a possibility for osteogenic genes to be up-regulated during adipogenic differentiation, as reported by Wall et al. [16]. One study showed that bone marrow-derived stem cells were able to maintain their osteogenic differentiation ability through 10-15 passages but not their adipogenic ability, which declined after serial passaging [17]. However, another study reported the adipogenic differentiation ability of bone marrow-derived stem cells was variable after clonal expansion [18]. In addition, it is still unclear whether ASC are able to maintain their differentiation ability in long-term culture.

Although adipose tissue is regarded as a waste by many people, studies have shown that it has many clinical applications, especially in cosmetic surgery. One of the most common is breast augmentation using lipo-injection technique, combining autologous adipose derived stem (stromal) cells (ASC) with fat [19]. Another common procedure is the intradermal injection of ASC in the treatment of skin aging, especially for wrinkles [20]. In order to be used clinically, millions of stem cells need to be harvested and injected or implanted into the patients. Therefore, they need to be expanded for an extended period of time to obtain a huge number of cells for clinical application. However, the effect of long-term culture on the ability of ASC to differentiate into adipogenic cells as well as their molecular activity is still relatively unknown. Furthermore, it is important to determine at which passage expression of these genes starts to alter. These data may be used as an indicator in ASC long-term differentiation. In this study, we analysed the expression levels of the adipogenic genes, osteogenic genes and stemness genes before and after adipogenic induction of ASC in extensive culture using quantitative PCR ( $q P C R$ ). The expression levels of these genes can reveal the molecular behaviour of ASC in the progression of adipogenic differentiation. The data can then be used to evaluate the efficiency of ASC to undergo adipogenic differentiation in longterm culture.

\section{Material and methods}

\section{Isolation and culture of human adipose- derived stem cells}

The ASC were harvested from human lipoaspirate tissue, which was obtained from 6 patients 
(age: 40-60 years old) undergoing intraoperative suction lipectomy at the Subang Jaya Medical Centre, Malaysia. The use of human tissue and cells for research purposes in this study was approved by Universiti Kebangsaan Malaysia Research and Ethical Committee (Project code: 02-01-02SF0290). The lipoaspirate tissue was digested using $0.3 \%$ Collagenase Type I (Sigma-Aldrich, St Louise, MO) for $2 \mathrm{~h}$ at $37^{\circ} \mathrm{C}$. The digested tissue was centrifuged at $1200 \mathrm{~g}$ for $10 \mathrm{~min}$. The supernatant was discarded and the cell pellet was washed using phosphate buffered saline (PBS) twice. The cells were cultured in Dulbecco's Modified Eagle Medium (DMEM)/Ham F12 medium (Invitrogen, Carlsbad, CA) supplemented with 10\% fetal bovine serum (FBS) (Fisher Scientific, USA), 1\% antibiotic-antimycotic (Invitrogen, Carlsbad, CA), 1\% Glutamax (Invitrogen) and 1\% vitamin C (Sigma-Aldrich). Cell cultures were incubated and maintained at $37^{\circ} \mathrm{C}$ with $5 \%$ carbon dioxide. They were fed every 3 days. The cells were grown to confluence after the initial plating $(P=0)$ and they were sub-cultured at $1: 4$ expansion under the same condition for an extensive culture period until passage 20. Once confluent (80-90\%), the adherent cells were detached using $0.125 \%$ trypsin-EDTA (Invitrogen) and they were either re-plated at 2000 to 2500 cells $/ \mathrm{cm}^{2}$ or used for experimental analysis. Cell viability and numbers at the time of passage were determined by trypan blue exclusion and haemocytometer cell counts. Cells cultured at passages 5, 10, 15 and 20 were used for analysis. Phase contrast images of cell morphology were captured at P5, P10, P15 and $\mathrm{P} 20$.

\section{In vitro adipose-derived stem cells adipogenic differentiation and morphological evaluation}

The adipogenic differentiation capability of cultured ASC at various passages (P5, P10, P15 and P20) was tested by using the adipogenic differentiation medium (Dulbecco's Modified Eagle Medium (DMEM)/Ham F12) (Invitrogen) supplemented with $10 \%(\mathrm{v} / \mathrm{v})$ fetal bovine serum (Invitrogen), 1\% (v/v) antibiotic antimycotic (Invitrogen), 1\% (v/v) Glutamax (Invitrogen), 1\% (v/v) vitamin C (Sigma-Aldrich), $200 \mu \mathrm{M}$ indomethacin (Sigma-Aldrich), $10 \mu \mathrm{M}$ insulin (Sigma-Aldrich), 0.5 mM 3-isobutyl-1-methylxanthine (IBMX) (Sigma-Aldrich) and $1 \mu \mathrm{M}$ dexamethasone (Sigma-Aldrich). Cells were seeded at 2500 cells/ $\mathrm{cm}^{2}$ in a $28 \mathrm{~mm}^{2}$ cell culture plate and maintained in a $5 \% \mathrm{CO}_{2}$ incubator (RS Biotech Galaxy Plus, Scotland, UK) at $37^{\circ} \mathrm{C}$ under $95 \%$ humidity for 3 weeks. The cell cultures were maintained in normal cell culture medium for 3 days before changing to the adipogenic differentiation medium. The two media were alternately changed every 3 days due to the toxicity of the adipogenic differentiation medium. The ASC underwent adipogenic induction for 3 weeks. Cultures were examined every day by using an inverted phase contrast microscope for monitoring cells' morphological changes. Photographs were taken to record the changes in cell morphology.

Adipogenic cells were identified by the formation of lipid droplets in the cell cytoplasm. The lipid droplets were stained red with Oil Red O staining (Sigma-Aldrich). After adipogenic induction, the cells were fixed with $10 \%(\mathrm{v} / \mathrm{v})$ formalin. The seeded cells were rinsed with distilled water thrice and they were dipped into $60 \%$ aqua triethyl phosphate solution. The cells were then stained with oil red $O$ for 15 min and rinsed with distilled water. The cells were then stained with Gill's haematoxylin and mounted with buffered glycerine. The stained cells were observed under the inverted contrast phase microscope (Olympus, Shinjuku-ku, Tokyo) for evaluation. The percentage of differentiated ASCs that produced lipid droplets was quantified using an image analyzer (Leica Microsystems, Wetzlar, Germany).

\section{Total RNA extraction}

Total RNA was extracted from the non-differentiated and differentiated ASCs cultured at P5, P10, P15 and P20. The RNA was homogenised and extracted using TRI reagent (Molecular Research Center, Cincinnati, OH). Chloroform was used for phase separation of the sample. Total RNA from the sample was precipitated by using isopropanol and polyacryl carrier (Molecular Research Center). The RNA extract was then centrifuged at $12000 \mathrm{~g}$ for $8 \mathrm{~min}$ at $4^{\circ} \mathrm{C}$. Seventy-five percent ethanol was used to wash the extract. RNase and DNase free distilled water (Invitrogen) was used to solubilise the RNA extract.

\section{cDNA synthesis}

The solubilised RNA extracts from ASCs cultured at P5, P10, P15 and P20 were used to synthesise cDNA by using SuperScript III First-Strand Synthesis SuperMix kit for two-step quantitative RT-PCR (Invitrogen). The synthesis was carried out according to the optimised protocol recommended by the manufacturer. The protocol conditions were $10 \mathrm{~min}$ at $23^{\circ} \mathrm{C}, 60 \mathrm{~min}$ at $42^{\circ} \mathrm{C}$ and $10 \mathrm{~min}$ at $94^{\circ} \mathrm{C}$. The synthesised CDNA was used to perform quantitative polymerase chain reaction ( $q P C R$ ) to determine the expression level of the stemness genes, adipogenic genes and osteogenic genes. 


\section{Quantitative polymerase chain reaction}

The non-differentiated and differentiated ASCs cultured at P5, P10, P15 and P20 were analysed for expression level of stemness genes, i.e. Sox2, Nanog3, Nestin, BST-1 (bone marrow stromal antigen 1) and Oct-4. Expression of adipogenic genes LPL, aP2 and PPAR- $\gamma$ as well as osteogenic genes BSP (bone sialoprotein), RUNX, ALP (alkaline phosphatase), OSP (osteopontin) and OSC (osteocalcin) was also evaluated. Primers for each gene were designed by using Primer 3 software based on the published GenBank database sequences. The PCR reaction was carried out using SYBR Green as the indicator in a Bio-Rad iCycler PCR machine. The reaction mixture consisted of iQ SYBR Supermix, forward and reverse primers (500 nM each), deionised water and $5 \mu$ l of cDNA. The reaction conditions were cycle 1 : step $195^{\circ} \mathrm{C}$ for 3 min (1 time), cycle 2: step $195^{\circ} \mathrm{C}$ for $10 \mathrm{~s}$ and step $261^{\circ} \mathrm{C}$ for $30 \mathrm{~s}$ (40 times) and cycle 3 for melting curve analysis. The expression level of each targeted gene was normalised to GAPDH as the housekeeping gene.

\section{Statistical analysis}

Data were analysed using SPSS 15.0 (SPSS Inc., Chicago, IL) and subjected to one-way analysis of variance with a Tukey post hoc test to determine significant differences $(p<0.05)$ in gene expression levels between passages P5, P10, P15 and P20. Data are presented as the mean \pm standard error of mean (SEM).

\section{Results}

Morphological and growth kinetic changes of adipose-derived stem cells in extensive culture

Extensive sub-cultivation altered the morphology and the growth kinetics of human ASC. The ASC has a fibroblast-like morphology and this feature was most obvious at early passages (P5) (Figure $1 \mathrm{~A}$ ). The cell morphology, i.e. the size and shape, were consistent with minimal alterations at passages P5 to P10 (Figure $1 \mathrm{~B}$ ). However, marked alterations in the cell morphology were observed at passages P15 to P20 (Figures $1 \mathrm{C}$ and $1 \mathrm{D}$ ). This altered morphology was indicated by the disappearance of the fibroblast-like feature and the appearance of large cells with expanded cytoplasmic volume. Passages beyond P20 could not be analysed as the cell growth became so protracted where the cells failed to reach $80-90 \%$ confluence even after 3 weeks in culture. The study on ASC growth rate showed no significant changes in the growth rate at P5 (1347 cells/cm²/day), P10 (2413 cells $/ \mathrm{cm}^{2} /$ day) and P15 (1645 cells/cm²/day) (Fig-
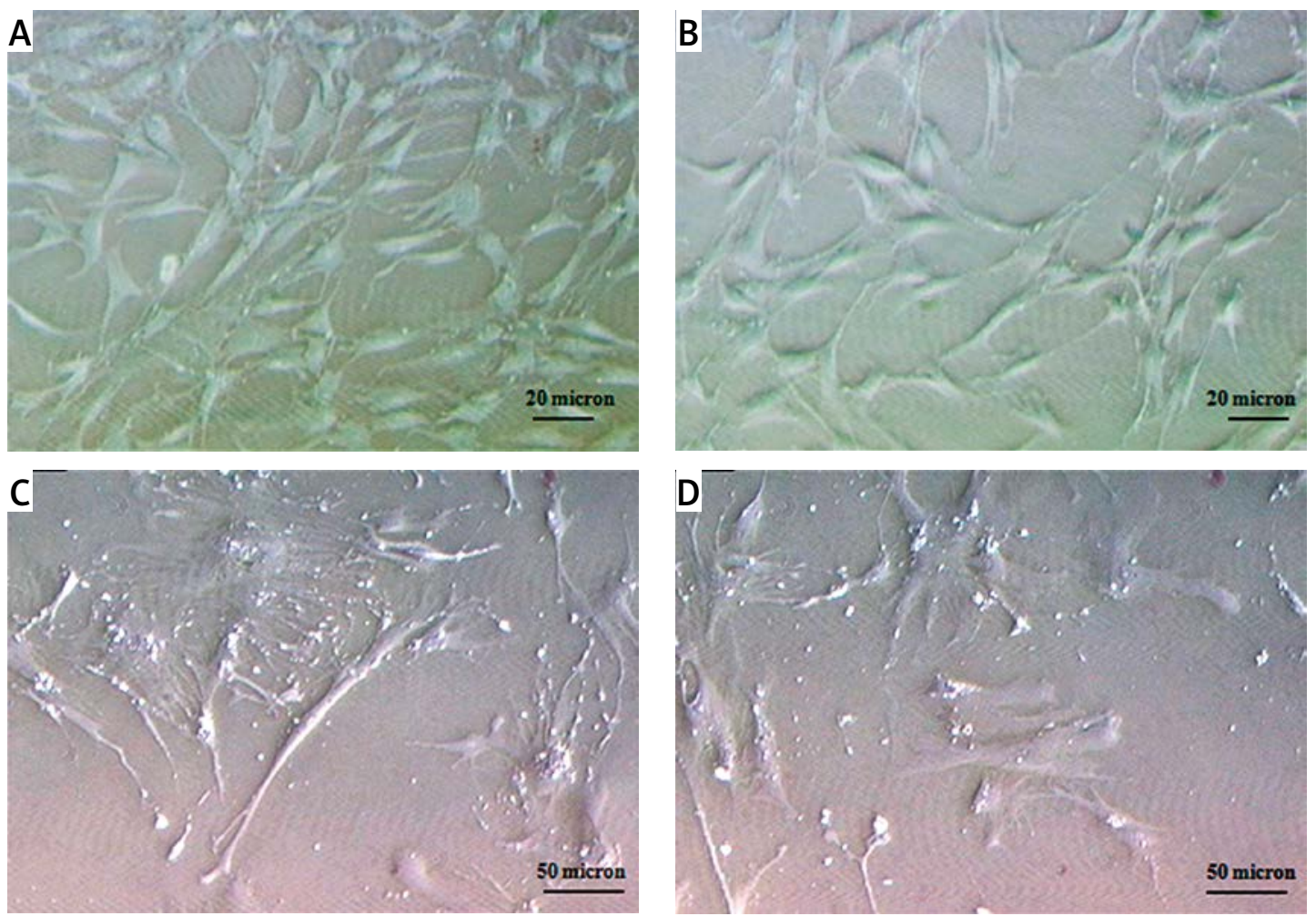

Figure 1. Representative cell morphology of ASC in extensive culture. A - Passage 5, B - Passage 10, C - Passage 15 and D - Passage 20. Magnification 200x 
ure 2). However, the decline in growth rate was significant $(p<0.05)$ at P20 (254 cells/ $\mathrm{cm}^{2} /$ day $)$ (Figure 2) compared to P10.

\section{Morphological changes of adipose-derived stem cells undergoing adipogenesis}

After 3 days in adipogenic medium, the shape of the cells changed from fibroblastic to a more spherical shape with 'ghost-like' features (Figure 3 A), which marked the start of adipogenesis. The hallmark of adipogenesis is the formation of lipid droplets where the appearance of lipid droplets can be observed in the cytoplasm of the cells from 7 days onwards after adipogenic induction. The formation of these lipid droplets was more prominent at an early passage (P5) with large and shiny droplets (Figures $3 \mathrm{~B}$ and $3 \mathrm{C}$ ) and less prominent at a later passage (P20) with more ghost-like features, which only pick up a small amount of the red stain after Oil Red $O$ staining (Figures $3 \mathrm{D}$ and $3 \mathrm{E}$ ). At P5, the percentage of differentiated ASC that produced lipid droplets was $22.5 \%$. At P10 and P15 the percentage of differentiated ASC was $20 \%$ and $10 \%$, respectively, while at P20, the percentage of differentiated ASC that produced lipid droplets was $6 \%$.

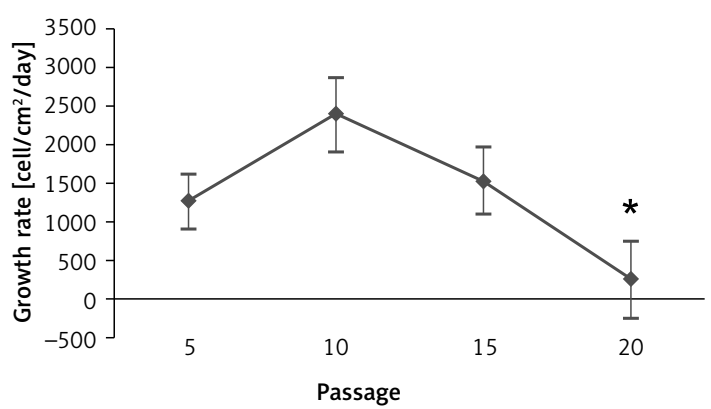

Figure 2. Growth rate (cell/ $/ \mathrm{cm}^{2} /$ day) of ASC in extensive culture from $\mathrm{P} 5$ to $\mathrm{P} 20$. The growth rate peaked at P10 but decreased significantly at P20

${ }^{*} p<0.05$ relative to $P 10$ by ANOVA post hoc Tukey test

After 21 days, most cells at P20 died as the cells at this passage cannot withstand the toxicity of adipogenic medium. Differentiated ASCs at P10 and P15 have similar features as P5.

\section{Adipogenic gene expression in extensive culture of adipose-derived stem cells}

The PPAR (Figure $4 \mathrm{~A}$ ) increased in expression after adipogenic induction at all passages. At P5, the PPAR expression level after induction increased
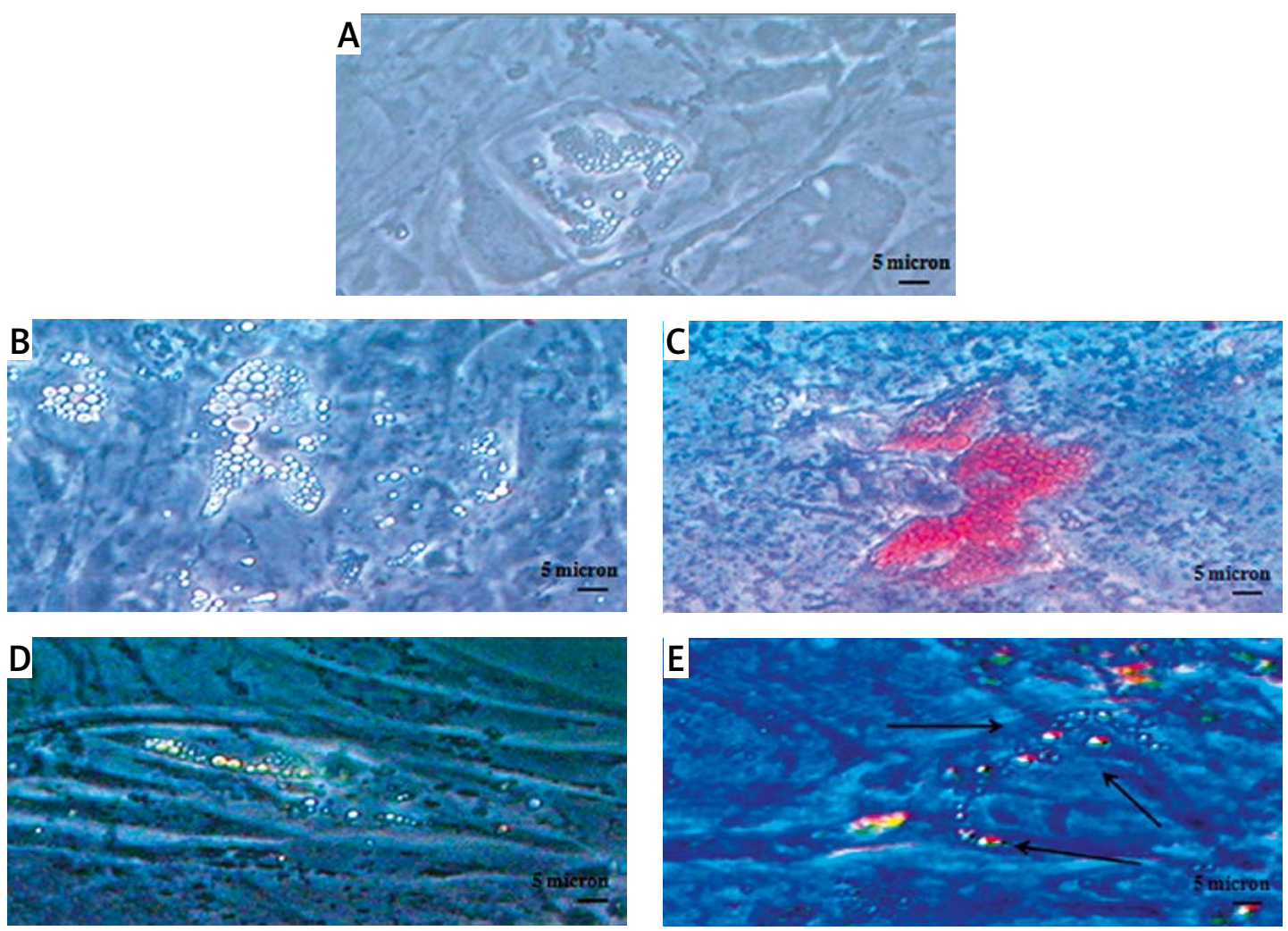

Figure 3. Morphology of adipogenic differentiation of ASC. A - The cells had a more 'ghost-like' feature which marked the beginning of adipogenesis. Morphology of ASC after 21 days' adipogenic induction at P5 (B) and P20 (D). Oil Red O staining of differentiated ASC at P5 (C) and P20 (E). Differentiated ASC at P10 and P15 have similar features as ASC at P5. Magnification 200x 
A

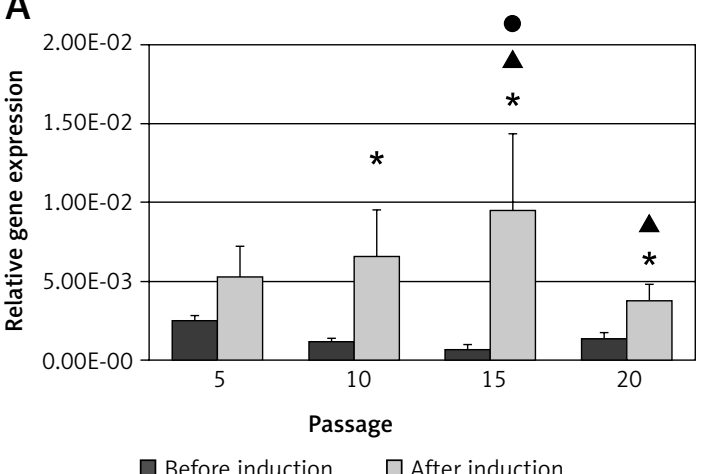

$\square$ Before induction $\square$ After induction

C

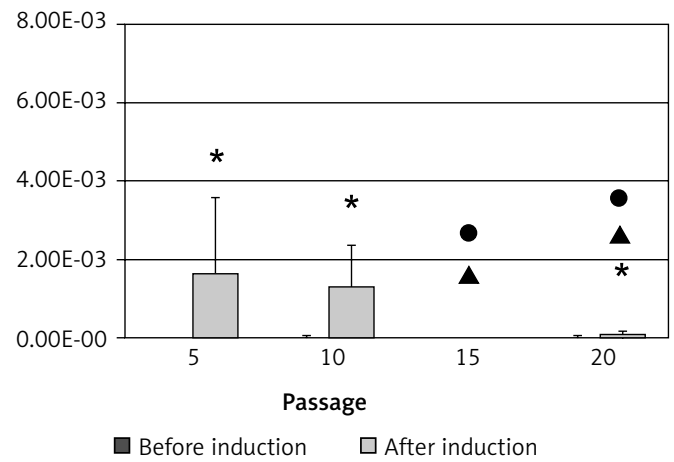

to $5.31 \mathrm{E}-03 \pm 1.94 \mathrm{E}-03$ compared to before induction. At P10, the PPAR expression level increased significantly $(p<0.05)$ to $6.62 \mathrm{E}-03 \pm 2.90 \mathrm{E}-03 \mathrm{com}$ pared to before induction. The expression also increased significantly at P15 and P20 $(p<0.05)$ from $7.05 \mathrm{E}-04 \pm 3.11 \mathrm{E}-04$ to $9.58 \mathrm{E}-03 \pm 4.74 \mathrm{E}-03$ and from $1.46 \mathrm{E}-03 \pm 3.22 \mathrm{E}-04$ to $3.75 \mathrm{E}-03 \pm 1.08 \mathrm{E}-03$, respectively, after induction. Among the entire induced group, PPAR expression level was significantly the highest at P15 $(p<0.05)$ compared to P5 and P10.

The LPL (Figure 4B) increased in expression after ASC induced in adipogenic medium at all passages. At P5, LPL increased from 2.60E-06 $\pm 1.54 \mathrm{E}-06$ to $3.56 \mathrm{E}-04 \pm 7.55 \mathrm{E}-04$, while at P10, LPL increased in expression from 3.18E-06 $\pm 2.49 \mathrm{E}-06$ to $1.62 \mathrm{E}-03 \pm 1.51 \mathrm{E}-03$. At $\mathrm{P} 15$ it increased significantly from $5.58 \mathrm{E}-07 \pm 3.23 \mathrm{E}-07$ to $1.70 \mathrm{E}-03$ $\pm 1.59 \mathrm{E}-03$. At P20 there was only a slight increase in expression of LPL from 3.89E-07 $\pm 2.25 \mathrm{E}-07$ to $4.89 \mathrm{E}-06 \pm 3.61 \mathrm{E}-06$. Among the entire induced group, P15 showed the highest expression level of LPL compared to other passages. It is statistically significant when compared to P10 $(p<0.05)$. In contrast, at P20, the expression level of LPL is the lowest and it is statistically significant $(p<0.05)$ when compared to P5 and P10.

aP2 (Figure $4 \mathrm{C}$ ) also increased in expression after adipogenic induction although not as high as PPAR and LPL. At P5 and P10, it increased significantly $(p<0.05)$ from $1.37 \mathrm{E}-05 \pm 5.12 \mathrm{E}-06$ to
B

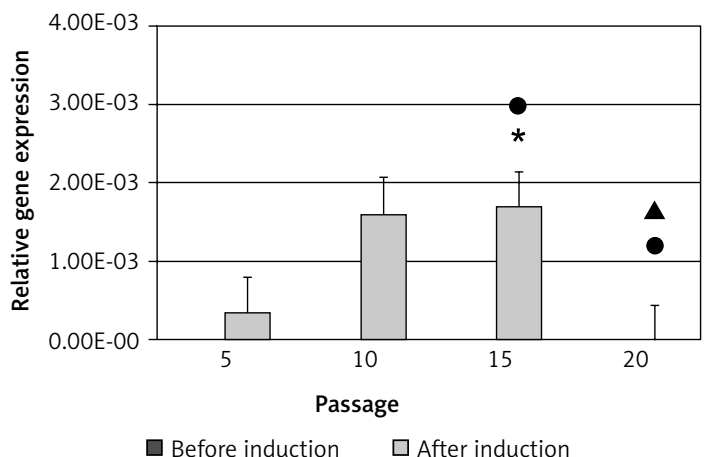

Figure 4. Relative changes in adipogenic gene expression levels in ASC at P5, P10, P15 and P20 before and after adipogenic induction $(n=6)$. A PPAR gene; $\mathbf{B}-\mathrm{LPL}$ gene and $\mathbf{C}-\mathrm{aP} 2$ gene expression level

${ }^{*} p<0.05$ relative to before induction by paired $t$-test, $\Delta_{p}<0.05$ relative to $P 5$ after induction by one-way ANOVA post hoc Tukey, $\bullet<0.05$ relative to $P 10$ after induction by one-way ANOVA post hoc Tukey

$1.66 \mathrm{E} 03 \pm 1.90 \mathrm{E}-03$ and from $7.19 \mathrm{E}-06 \pm 2.16 \mathrm{E}-06$ to $1.31 \mathrm{E}-03 \pm 1.07 \mathrm{E}-03$ respectively. At $\mathrm{P} 15$, there was slight increase in aP2 expression from 8.07E$06 \pm 3.99 \mathrm{E}-06$ to $1.28 \mathrm{E}-05 \pm 5.95 \mathrm{E}-06$, while at $\mathrm{P} 20$, the aP2 expression level increased significantly $(p<0.05)$ from $5.81 \mathrm{E}-06 \pm 3.49 \mathrm{E}-06$ to $1.04 \mathrm{E}-04$ $\pm 7.13 \mathrm{E}-05$. Among all the induced groups, aP2 expression was the highest at P5 and the lowest at P15 compared to other passages. The aP2 expression at P15 was significantly decreased $(p<0.05)$ compared to P5 and P10. While at P20, aP2 was also significantly decreased $(p<0.05)$ compared to P5 and P10.

\section{Osteogenic gene expression in adipose- derived stem cells after adipogenesis}

Apart from adipogenic genes, osteogenic genes, i.e. ALP, OSP, BSP, OSC and RUNX, were also evaluated under adipogenic differentiation. There were no significant changes in the osteogenic gene expression levels during adipogenic induction with the exception of OSP. These genes showed low expression levels during adipogenic induction with no significant difference compared to before induction. The ALP showed a modest increase in expression at P10, P15 and P20 compared to before induction (Figure $5 \mathrm{~A}$ ), while OSP (Figure 5 B) showed a modest increase in expression at P5, P10 and P15 but there was a significant increase at P20 $(p<0.05)$ compared 
A

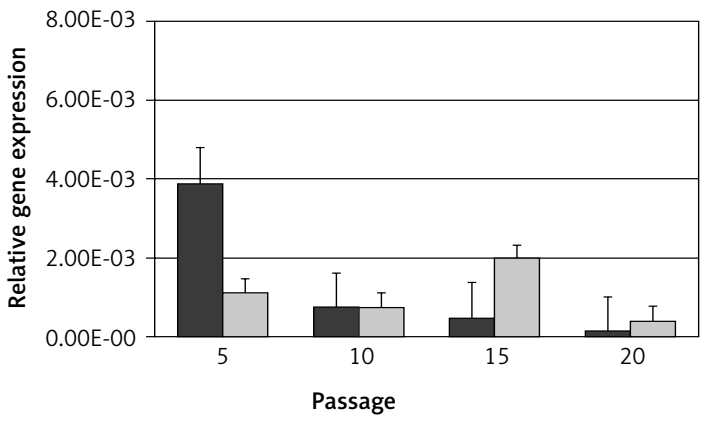

$\square$ Before induction $\square$ After induction

C

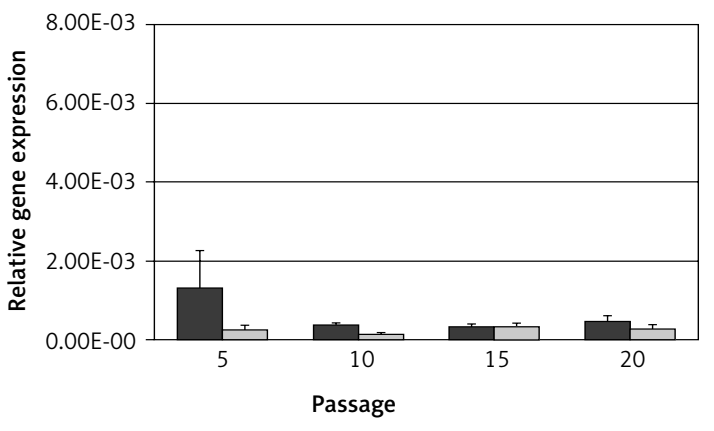

$\square$ Before induction $\square$ After induction

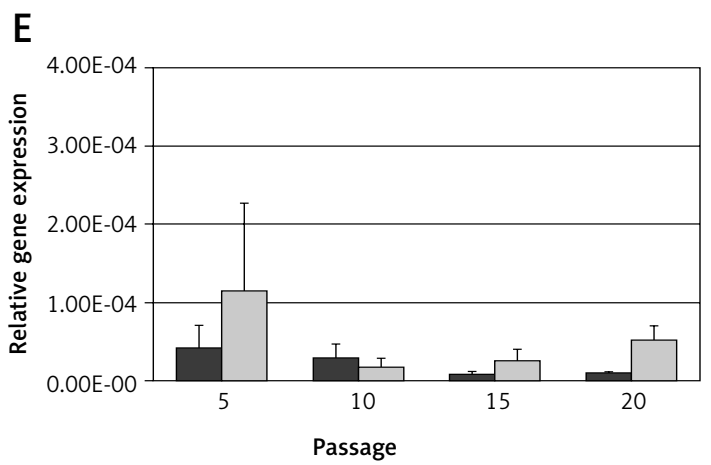

$\square$ Before induction $\quad \square$ After induction

to before induction, from $4.47 \mathrm{E}-04 \pm 1.44 \mathrm{E}-04$ to $2.66 \mathrm{E} 04 \pm 1.04 \mathrm{E}-04$. The OSC (Figure $5 \mathrm{C}$ ) showed a very low expression at all passages compared to before induction. RUNX (Figure $5 \mathrm{D}$ ) and BSP (Figure $5 \mathrm{E}$ ) showed a modest increase at P15 and P20 after induction.

\section{Stemness gene expression in adipose-} derived stem cells after adipogenesis

Stemness gene expression was also studied before and after adipogenic induction at P5, P10, P15 and P20. Nanog3 (Figure 6 A) showed a significant increase $(p<0.05)$ in expression levels after adipogenic induction at P15 (before: 3.71E$05 \pm 7.05 \mathrm{E}-06$; after: 5.70E-05 $\pm 1.61 \mathrm{E}-05)$ and P20 (before: 4.93E-05 $\pm 8.09 \mathrm{E}-06$; after: 1.05E-04
B

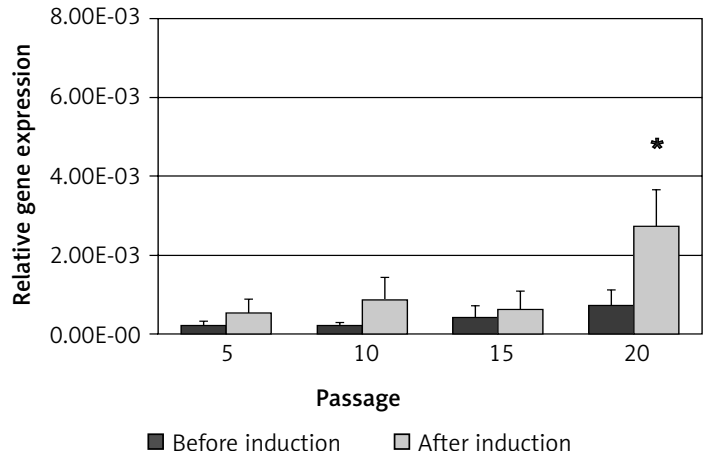

D

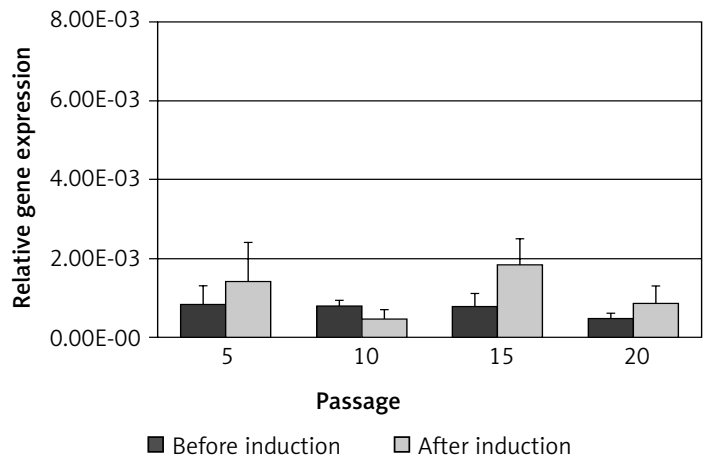

Figure 5. Relative changes in osteogenic gene expression levels in ASC at P5, P10, P15 and P20 before and after adipogenic induction $(n=6)$. A - ALP gene, $\mathrm{B}$ - OSP gene, C - OSC gene, D - RUNX gene and $\mathbf{E}-$ BSP gene expression level

${ }^{*} p<0.05$ relative to before induction by paired $t$-test

$\pm 2.91 \mathrm{E}-05$ ). Sox2 (Figure 6 B), Nestin (Figure 6 C) and Oct4 (Figure 6 E) showed no significant changes in expression levels at all the passages. However, BST1 (Figure 6 D) showed a significant decrease $(p<0.05)$ in expression at P5 (before: 2.64E-02 $\pm 8.27 \mathrm{E}-03$; after: 7.03E-03 $\pm 1.73 \mathrm{E}-03$ ), P10 (before: $2.54 \mathrm{E}-02 \pm 7.82 \mathrm{E}-03$; after: $1.53 \mathrm{E}-03$ $\pm 2.64 \mathrm{E}-04$ ), P15 (before: $1.86 \mathrm{E}-02 \pm 1.73 \mathrm{E}-03$; after: $1.60 \mathrm{E}-03 \pm 5.12 \mathrm{E}-04$ ) and P20 (before: $1.81 \mathrm{E}-02$ $\pm 7.13 \mathrm{E}-03$; after: 1.01E-02 $\pm 1.09 \mathrm{E}-03)$ after adipogenic induction.

\section{Discussion}

Adipogenic cells can be easily distinguished by alteration in cell shape where the fibroblastic cells changes to spherical shape cells after induction [9]. From the evaluation of morphology 
A

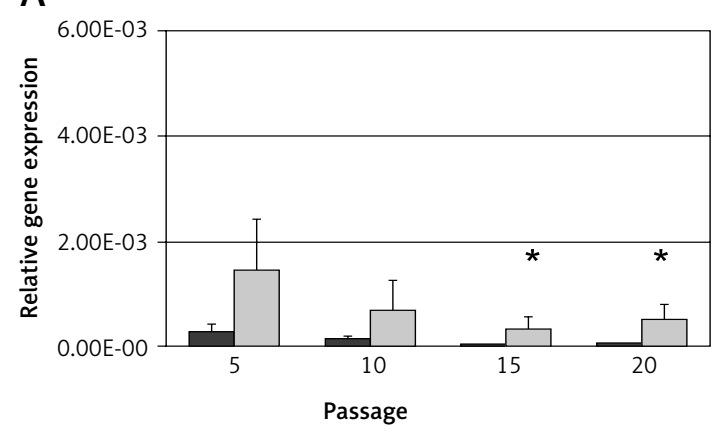

$\square$ Before induction $\quad \square$ After induction

C

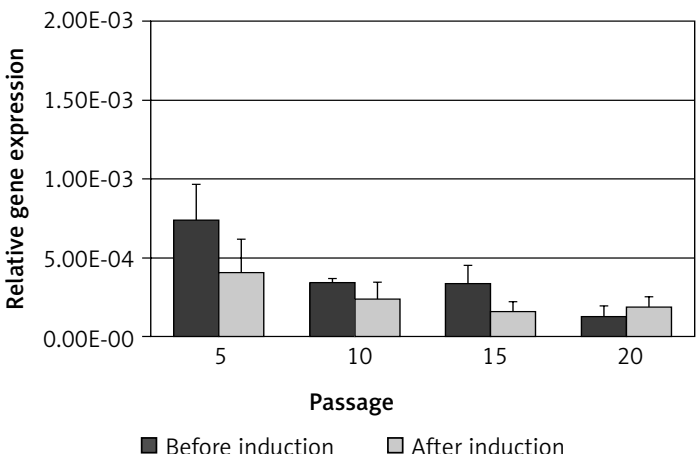

E

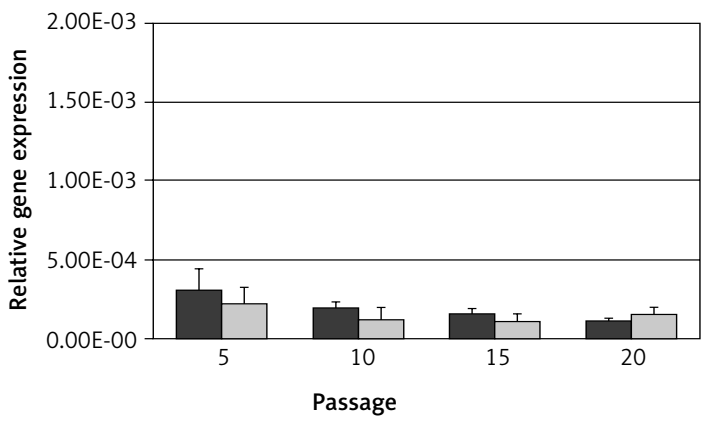

$\square$ Before induction $\quad \square$ After induction

and adipogenic gene expression levels, the ASC were found to be able to differentiate into adipogenic cells up to P20. We did not study passages beyond P2O since the cells became protracted and unable to reach the seeding density that was needed for adipogenic differentiation. In addition, some cells at P20 have already shown senescence characteristics and inability to withstand the toxicity of the adipogenic medium. Some of these cells died before the formation of lipid droplets could occur.

At passages 5, 10 and 15, PPAR- $\gamma$ and LPL expression levels increased significantly, while aP2 expression levels only increased up to P10 and to a lesser extent at P15 and P20. This showed that ASC were able to differentiate into mature adipogenic cells up to P10 and to a lesser extent
B

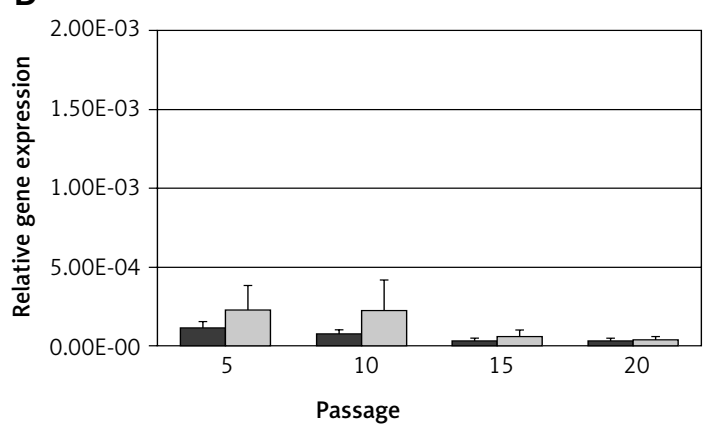

$\square$ Before induction $\quad \square$ After induction

D

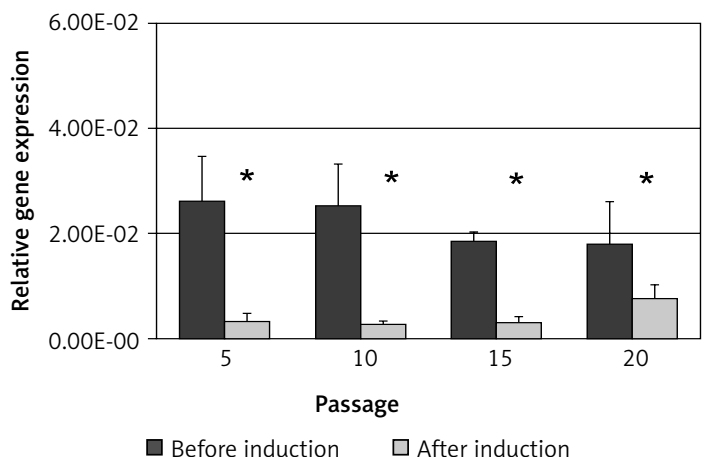

$\square$ Before induction $\square$ After induction

Figure 6. Relative changes in stemness gene expression levels in ASC at P5, P10, P15 and P20 before and after adipogenic induction $(n=6)$. A - Nanog gene, B - Sox 2 gene, $\mathbf{C}$ - Nestin gene, D - BST1 gene and E - Oct4 gene expression level

* $p<0.05$ relative to before induction by paired $t$-test

at P15. This was also supported by the decline in the formation of oil droplets at later passages. In addition, aP2 was the first to decline (starting from P10) in expression in extensive culture. The aP2 gene plays an important role in the development of metabolic syndrome and the coordination of cholesterol trafficking as well as inflammatory activity $[13,14]$. This indicates that in extensive culture, ASC may lose their adipogenic differentiation ability starting from P10. Izadpanah et al. also reported that ASCs significantly lost adipogenic potential but this occurred after P20 [21]. However, Wall et al. reported a similar result that ASCs were only capable of undergoing adipogenesis up to P10 [16]. In this study, we found that cells after P10 lose their ability to differentiate into adipogenic cells, which is in contrast to 
Izadpanah et al. In addition, the aP2 gene may be used as an indicator for adipogenic differentiation efficiency.

We also found that osteogenic genes, i.e. ALP, OSC, OSP, BMP and RUNX, were expressed during adipogenic induction but the expression levels were low and there was no significant change in their expression level after induction with the exception of OSP, which showed a significant increase at P20 after induction. While it is tempting to claim that there is a possibility of osteogenic dominance during adipogenic differentiation at later passage as reported by Wall et al. [16], the increase of OSP expression at P20 alone is not enough to justify that notion. Furthermore, there was no calcium deposition observed in our morphological study as reported by Wall et al. [16]. On the other hand, certain osteogenic genes may not be exclusively involved in the development of osteoblasts but could also play a role in the development of other cell types, e.g. the ALP gene, which was reported to be involved in intracellular lipid accumulation in 3T3-L1 cells and human preadipocytes [22]. In addition, adipogenic and osteogenic differentiation were suggested as parallel events [23] and not inverse events as found in another study [24]. However, data from our quantitative PCR analysis did not indicate that adipogenic and osteogenic differentiation are parallel events.

Most of the stemness genes with the exception of Nanog3 showed either a decrease or no significant changes in expression levels after adipogenic induction, which showed that the ASC may have terminally differentiated into specific cells, in this case adipocytes, thus reducing the number of stem cells in the whole cell population. Nanog3 showed an increase in expression at all passages after adipogenic induction with a significant increase at P15 and P20. However, when compared among passages, the expression of Nanog3 after adipogenic induction decreased at P15 and P20 compared to P5 and P10. Nanog supports stem cell self-renewal as well as maintenance of ESC pluripotency $[25,26]$. It interacts with Oct4 and Sox2 to induce self-renewal and differentiation [27]. However, increase or over-expression of $\mathrm{Na}$ nog has been found to slow down the progression of adipogenesis [28]. This suggests that increase in Nanog3 expression may also contribute to the decrease in the differentiation ability of ASC into mature adipocytes, especially at later passages in the current study. Nanog3 can become an indicator to evaluate the capacity of stem cells to differentiate into adipogenic cells.

In conclusion, the ASCs' ability to differentiate into mature adipogenic cells started to decrease in culture higher than P10 according to quantita- tive PCR and morphological analysis. Therefore, ASC culture at later passages (P15 and P20) is not suitable for adipogenic differentiation. The expression of stemness genes showed that the stem cell pool in the whole cell population decreased due to cell differentiation. The data on osteogenic gene expression suggested that the processes of osteogenesis and adipogenesis were not parallel events.

\section{Acknowledgments}

We thank the Ministry of Science, Technology \& Innovation of Malaysia (MOSTI) for financial support. Grant no: 02-01-02 SF0290.

\section{References}

1. Zuk PA, Zhu M, Ashjian P, et al. Human adipose tissue is a source of multipotent stem. Mol Biol Cell 2002; 13: 4279-95.

2. Liao D, Gong P, Li A, Tan Z, Yuan Q. Co-culture with Schwann cells is an effective way for adipose-derived stem cells neural transdifferentiation. Arch Med Sci 2010; 6: 145-51.

3. Kashani IR, Zaminy A, Barbarestani M, et al. In vitro osteogenesis of rat adipose-derived stem cells: comparison with bone marrow stem cells. Arch Med Sci 2009; 5: 149-55.

4. Guilak F, Lott KE, Awad HA, et al. Clonal analysis of the differentiation potential of human adipose-derived adult stem cells. J Cell Physiol 2006; 206: 229-37.

5. Leong TW, Hutmacher DW, Chew FT, Lim TC. Viability and adipogenic potential of human adipose tissue processed cell population obtained from pump-assisted and syringe-assisted liposuction. J Derm Sci 2004; 37: 169-76.

6. Ko KS, Lee IW, Joo WI, et al. Differentiation of human adult adipose derived stem cell in vitro and immunohistochemical study of adipose derived stem cell after intracerebral transplantation in rats. J Korean Neurosurg Soc 2007; 42: 118-24.

7. Ntambi JM, Kim YC. Adipocyte differentiation and gene expression. J Nutr 2000; 130: 3122S-6S.

8. Gregoire FM, Smas CM, Sul HS. Understanding adipocyte differentiation. Physiol Rev 1998; 78: 783-809.

9. Gregoire FM. Adipocyte differentiation: from fibroblast to endocrine cell. Exp Biol Med 2001; 226: 997-1002.

10. Michalik L, Auwerx J, Berger JP, et al. International union of pharmacology. LXI. Peroxisome proliferator-activated receptors. Pharmacol Rev 2006; 58: 726-41.

11. Mead JR, Irvine SA, Ramji DP. Lipoprotein lipase: structure, function, regulation and role in disease. J Mol Med 2002; 80: 753-69.

12. Michaud SE, Renier G. Direct regulatory effect of fatty acids on macrophage lipoprotein lipase: potential role of PPARs. Diabetes 2001; 50: 660-6.

13. Makowski L, Hotamisligil GS. Fatty acid binding proteins-the evolutionary crossroads of inflammatory and metabolic responses. J Nutr 2004; 134: 2464S-8S.

14. Makowksi L, Brittingham KC, Reynolds JM, Suttles J, Hotamisligil GS. The fatty acid-binding protein aP2, coordinates macrophage cholesterol trafficking and inflammatory activity. J Biol Chem 2005; 280: 12888-95.

15. Wan Kamarul Zaman WS, Makpol S, Santhapan S, Chua KH. Stemness gene expression profile of human 
adipose derived stem cells in long-term culture. Med J Malaysia 2008; 63 (Supplement A): 61-2.

16. Wall ME, Bernacki SH, Loboa EG. Effects of serial passaging on the adipogenic and osteogenic differentiation potential of adipose-derived human mesenchymal stem cells. Tissue Eng 2007; 13: 1291-8.

17. DiGirolamo CM, Stokes D, Colter D, Phinney DG, Class R, Prockop DJ. Propagation and senescence of human marrow stromal cells in culture: a simple colony-forming assay identifies samples with the greatest potential to propagate and differentiate. $\mathrm{Br} J$ Haematol 1999; 107: 275-81.

18. Halleux C, Sottile V, Gasser JA, Seuwen K. Multi-lineage potential of human mesenchymal stem cells following clonal expansion. J Musculoskel Neuron Interact 2001; 2: 71-6.

19. Yoshimura K, Sato K, Aoi N, Kurita M, Hirohi T, Harii K. Cell-assisted lipotransfer for cosmetic breast augmentation: supportive use of adipose-derived stem/stromal cells. Aesth Plast Surg 2008; 32: 48-55.

20. Park BS, Jang KA, Sung JH, et al. Adipose-derived stem cells and their secretory factors as a promising therapy for skin aging. Dermatol Surg 2008; 34: 1323-6.

21. Izadpanah R, Trygg C, Patel B, et al. Biologic properties of mesenchymal stem cells derived from bone marrow and adipose tissue. J Cellular Biochem 2006; 99: 1285-97.

22. Ali AT, Penny CB, Paiker JE, Psaras G, Ikram F, Crowther NJ. The relationship between alkaline phosphatase activity and intracellular lipid accumulation in murine 3T3-L1 cells and human preadipocytes. Anal Biochem 2006; 354: 247-54.

23. Ponce ML, Koelling S, Kluever A, et al. Coexpression of osteogenic and adipogenic differentiation markers in selected subpopulations of primary human mesenchymal progenitor cells. J Cell Biochem 2008; 104: 1342-55.

24. Jaiswal RK, Jaiswal N, Bruder SP, Mbalaviele G, Marshak DR, Pittenger MF. Adult human mesenchymal stem cell differentiation to the osteogenic or adipogenic lineage is regulated by mitogen-activated protein kinase. J Biol Chem 2000; 275: 9645-52.

25. Chambers I, Colby D, Robertson M, et al. Functional expression cloning of Nanog, a pluripotency sustaining factor in embryonic stem cells. Cell 2003; 113: 643-55.

26. Mitsui K, Tokuzawa Y, Itoh H, et al. The homeoprotein Nanog is required for maintenance of pluripotency in mouse epiblast and ES cells. Cell 2003; 113: 631-42.

27. Rodda DJ, Chew JL, Lim LH, et al. Transcriptional regulation of Nanog by OCT4 and SOX2. J Biol Chem 2005; 280: 24731-7.

28. Liu TM, Wu YN, Guo XM, Hui JHP, Lee EH, Lim B. Effects of ectopic Nanog and Oct4 overexpression on mesenchymal stem cells. Stem Cell Dev 2009; 18: 1013-22. 